\title{
Ralf Haselhuhn
}

\section{Photovoltaik}

Gebäude liefern Strom

7., vollständig überarbeitete Auflage

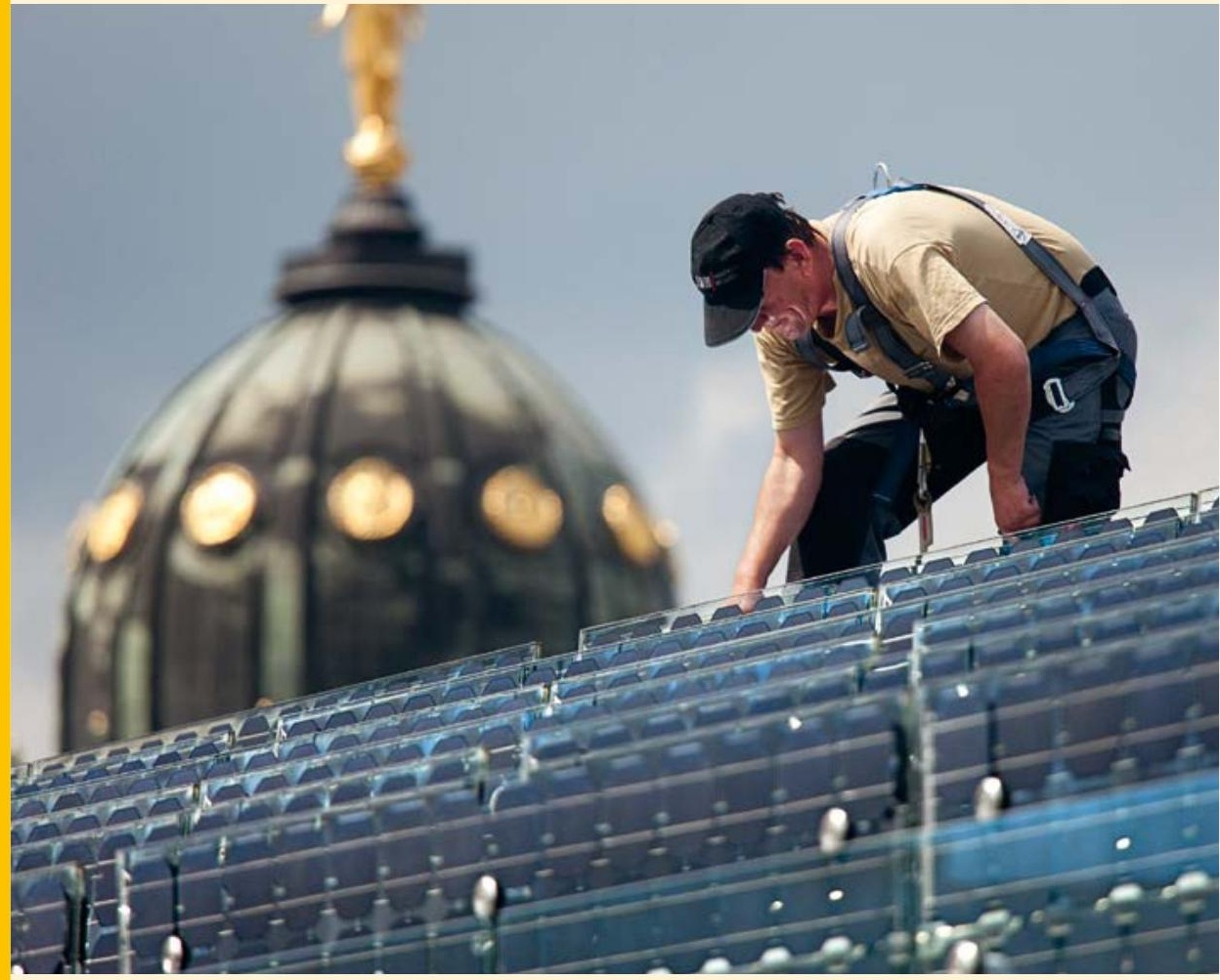

Fraunhofer IRB Verlag 
BINE-Fachbuch

Ralf Haselhuhn

Photovoltaik 
BINE-Fachbuch

\section{Photovoltaik}

7., vollständig überarbeitete Auflage

Der Autor:

Ralf Haselhuhn 
BINE Informationsdienst berichtet über Themen der Energieforschung: Neue Materialien, Systeme und Komponenten, innovative Konzepte und Methoden. BINE-Leser werden so über Erfahrungen und Lerneffekte beim Einsatz neuer Technologien in der Praxis informiert. Denn erstklassige Informationen sind die Grundlage für richtungsweisende Entscheidungen, sei es bei der Planung energetisch optimierter Gebäude, der Effizienzsteigerung industrieller Prozesse oder bei der Integration erneuerbarer Energien in bestehende Systeme.

BINE Informationsdienst ist ein Service von FIZ Karlsruhe und wird vom Bundesministerium für Wirtschaft und Technologie (BMWi) gefördert.

Für weitere Fragen steht Ihnen zur Verfügung:

Gerhard Hirn (Redaktion)

BINE Informationsdienst, FIZ Karlsruhe, Büro Bonn

Kaiserstraße 185-197, 53113 Bonn

Tel. +49 228923 79-0, E-Mail: bine@fiz-karlsruhe.de, www.bine.info

Bibliografische Information der Deutschen Nationalbibliothek:

Die Deutsche Nationalbibliothek verzeichnet diese Publikation in der Deutschen Nationalbibliografie; detaillierte bibliografische Daten sind im Internet über www.dnb.de abrufbar.

ISBN (Print): 978-3-8167-8737-2 I ISBN (E-Book): 978-3-8167-8738-9

Layout: Dietmar Zimmermann I Umschlaggestaltung: Martin Kjer I Herstellung: Tim Oliver Pohl

Satz: Mediendesign Späth GmbH, Birenbach I Druck: DZA Druckerei zu Altenburg GmbH, Altenburg

Alle Rechte vorbehalten.

Dieses Werk ist einschließlich aller seiner Teile urheberrechtlich geschützt. Jede Verwertung, die über die engen Grenzen des Urheberrechtsgesetzes hinausgeht, ist ohne schriftliche Zustimmung des Fraunhofer IRB Verlages unzulässig und strafbar. Dies gilt insbesondere für Vervielfältigungen, Übersetzungen, Mikroverfilmungen sowie die Speicherung in elektronischen Systemen.

Die Wiedergabe von Warenbezeichnungen und Handelsnamen in diesem Buch berechtigt nicht zu der Annahme, dass solche Bezeichnungen im Sinne der Warenzeichen- und Markenschutz-Gesetzgebung als frei zu betrachten wären und deshalb von jedermann benutzt werden dürften.

Sollte in diesem Werk direkt oder indirekt auf Gesetze, Vorschriften oder Richtlinien (z. B. DIN, VDI, VDE) Bezug genommen oder aus ihnen zitiert werden, kann der Verlag keine Gewähr für Richtigkeit, Vollständigkeit oder Aktualität übernehmen. Es empfiehlt sich, gegebenenfalls für die eigenen Arbeiten die vollständigen Vorschriften oder Richtlinien in der jeweils gültigen Fassung hinzuzuziehen.

Titelbild: Paul Langrock, paul-langrock.de

(c) by FIZ Karlsruhe

Verlag und Vertrieb:

Fraunhofer IRB Verlag

Fraunhofer-Informationszentrum Raum und Bau IRB

Nobelstraße 12, 70569 Stuttgart

Telefon +49711970-2500

Telefax $+49711970-2508$

irb@irb.fraunhofer.de

www.baufachinformation.de

Hinweis zu den Abbildungen: Soweit nachfolgend keine anderen Quellen genannt werden, stammen die Abbildungen von den Autoren. 


\section{Inhaltsverzeichnis}

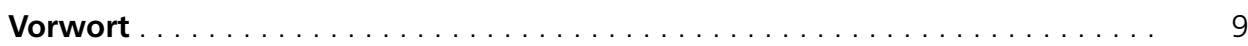

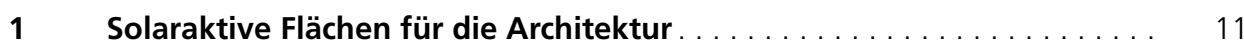

1.1 Gebäude mit Solarmodulen gestalten . . . . . . . . . . . . . . . . . . 11

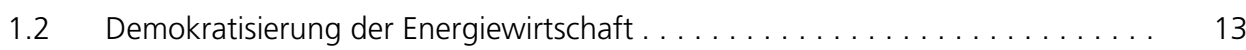

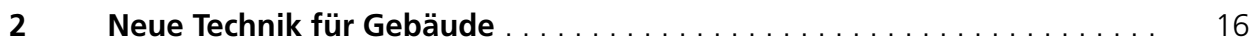

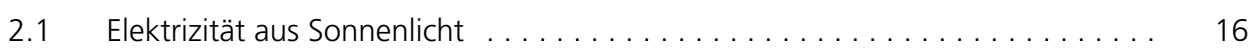

2.2 Die Solarzelle . . . . . . . . . . . . . . . . . . . . . . . . 17

2.3 Verschiedene Solarzellentypen . . . . . . . . . . . . . . . . . . . . 19

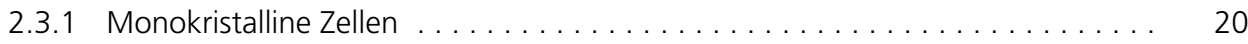

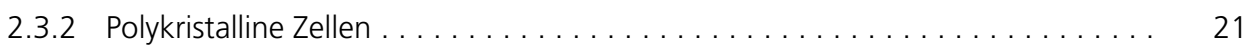

2.3 .3 Dünnschichtzellen ........................... 22

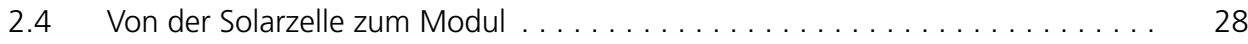

2.5 Speichersysteme ergänzen die Photovoltaikanlage $\ldots \ldots \ldots \ldots \ldots \ldots \ldots \ldots$

$3 \quad$ Am Anfang steht die Planung . . . . . . . . . . . . . . . 37

$3.1 \quad$ Wie viel Energie liefert die Sonne? . . . . . . . . . . . . . . . . . . 37

3.2 Vorbereitung und Gebäudebegutachtung. . . . . . . . . . . . . . 41

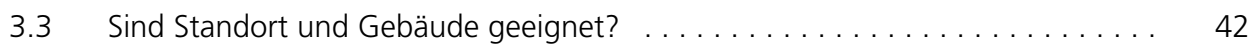

3.4 Verschattungseffekte vermeiden. . . . . . . . . . . . . . . . . 43

3.5 Anlage und Komponenten richtig dimensionieren . . . . . . . . . . . . . 46

3.5.1 Leistung, Flächenbedarf und Wirkungsgrad . . . . . . . . . . . . . . . . 46

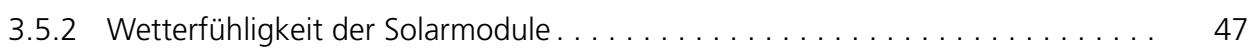

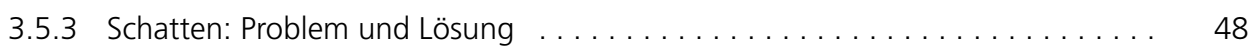

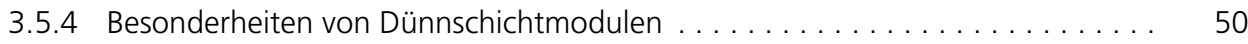

3.5.5 Verschaltung der Module zum Solargenerator . . . . . . . . . . . . . . . 52

3.6 Der Wechselrichter als Anlagenzentrale . . . . . . . . . . . . . . . . . 53

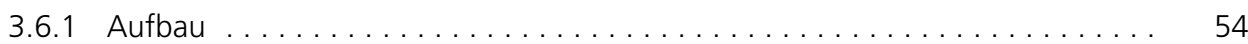

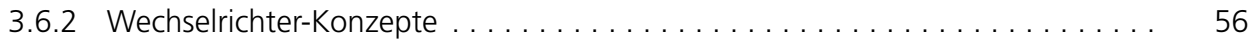

3.6.3 Optimale Performance - Europäischer Wirkungsgrad . . . . . . . . . . . . . . 57

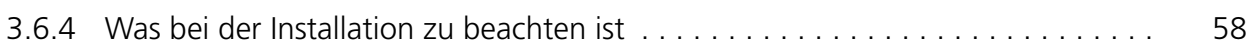

3.6.5 Solargenerator und Wechselrichter aufeinander abstimmen . . . . . . . . . 58

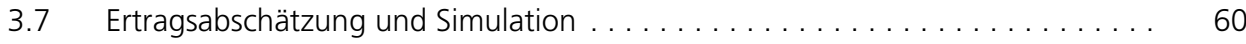

3.8 Planung von PV-Systemen zum Eigenverbrauch . . . . . . . . . . . . . 62

3.9 Checkliste zur erfolgreichen Planung . . . . . . . . . . . . . . . 68 


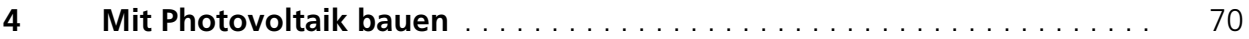

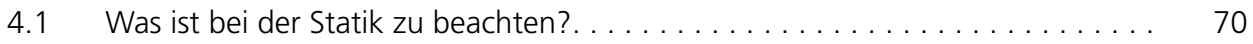

$4.2 \quad$ Montage auf dem Dach . . . . . . . . . . . . . . . . . . . . . 71

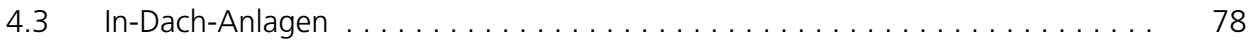

4.4 Aufgeständerte Systeme für das Flachdach $\ldots \ldots \ldots \ldots \ldots \ldots \ldots \ldots \ldots . \quad 81$

4.5 Montage an der Fassade. . . . . . . . . . . . . . . . . . . 84

4.5.1 Fassaden für die Photovoltaik-Integration $\ldots \ldots \ldots \ldots \ldots \ldots \ldots \ldots \ldots \ldots$

4.5 .2 Module mit linienförmigen Halterungen $\ldots \ldots \ldots \ldots \ldots \ldots \ldots \ldots \ldots$

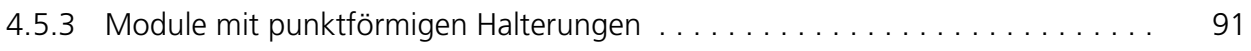

4.5 .4 Module als Sonnenschutz . . . . . . . . . . . . . . . . . . . . . 91

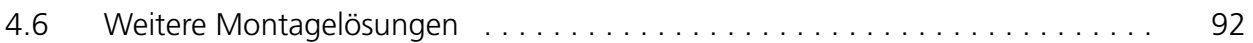

4.6.1 Wintergärten, Erschließungszonen und bauliche Anlagen . . . . . . . . . . 92

4.6 .2 Freiflächen-Anlagen . . . . . . . . . . . . . . . . . . . 94

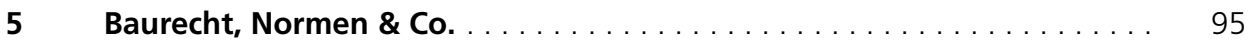

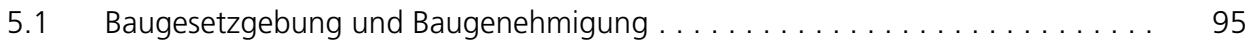

5.2 Bauordnung, Bauregeln, Bauprodukte und DIBt-Hinweispapier für Solar-

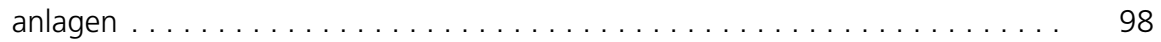

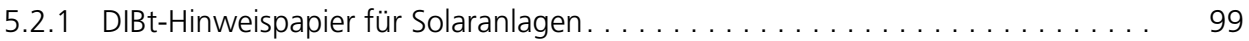

5.2 .2 Statik-Normen . . . . . . . . . . . . . . . . . . . . . . . . 101

5.3 Brandschutz ................................. 103

5.3.1 Brandschutzanforderungen der Bauordnung. . . . . . . . . . . . . . . 103

5.3.2 Anforderungen für die Brandbekämpfung . . . . . . . . . . . . . . . . 104

5.3.3 Weitere Anforderungen für den Brandschutz . . . . . . . . . . . . . . . 106

5.4 Photovoltaik als elektrische Anlage ... . . . . . . . . . . . . . . . . . 108

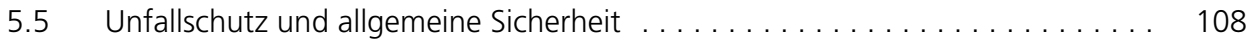

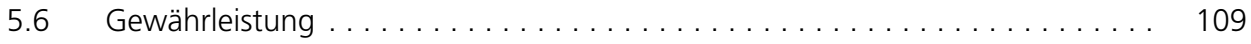

$6 \quad$ Elektrische Installation und Inbetriebnahme . . . . . . . . . . . . 110

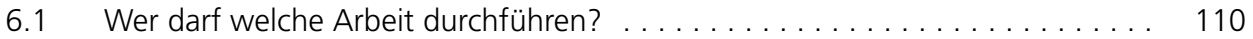

6.2 Der Generatoranschlusskasten . . . . . . . . . . . . . . . . . . . . . 111

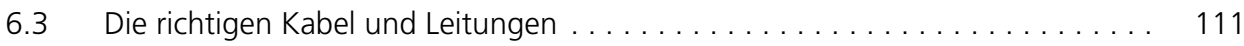

6.4 Schutz vor Fehlerströmen . . . . . . . . . . . . . . . . . . . . . . . 113

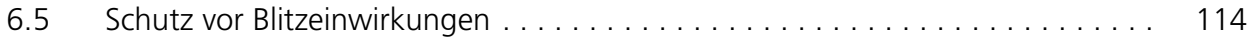

6.6 Geltende Regelungen für den Netzanschluss $\ldots \ldots \ldots \ldots \ldots \ldots \ldots \ldots \ldots$

6.7 Abnahme und Inbetriebnahme . . . . . . . . . . . . . . . . . . 121

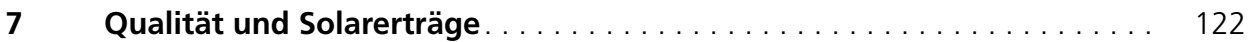

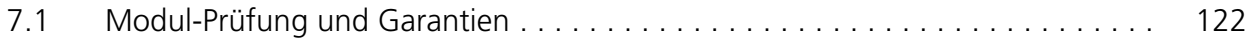

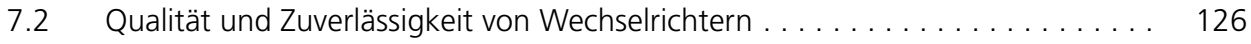




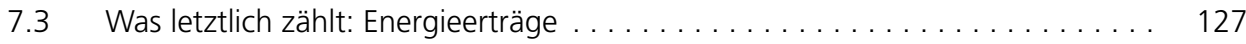

7.4 Ertragssicherung durch Betriebsdatenerfassung und Anlagenüberwachung . . . 128

7.5 Wartung und Instandhaltung . . . . . . . . . . . . . . . . . . . . . 129

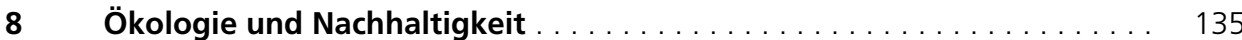

$8.1 \quad$ Energieversorgung - heute und morgen $\ldots \ldots \ldots \ldots \ldots \ldots \ldots \ldots \ldots$

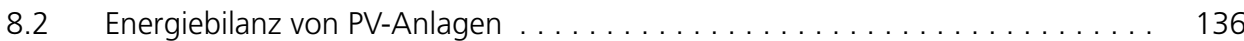

$9 \quad$ Kosten und Erlöse . . . . . . . . . . . . . . . . . . . . . . . . . . . . . . . . . 140

9.1 Anschub durch das Erneuerbare-Energien-Gesetz . . . . . . . . . . . . . . . . . 140

9.2 Investitionskosten dominieren die Wirtschaftlichkeit . . . . . . . . . . . . 145

9.3 Ergänzende öffentliche Fördermittel . . . . . . . . . . . . . . . . . . . . . 147

9.4 Steuerliche Nebenwirkungen . . . . . . . . . . . . . . . . . . . . . . . . . . . . . 148

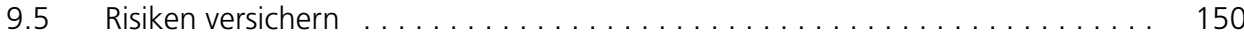

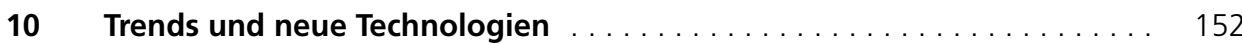

10.1 Neue Zelltechnologien . . . . . . . . . . . . . . . . . . . . . . . . . . . 152

10.2 Trends bei Solarmodulen. . . . . . . . . . . . . . . . . . . . . . . . . . . . . . . . 154

10.3 Neue Wechselrichter- und Anlagenkonzepte . . . . . . . . . . . . . . . . . 156

10.4 Auf dem Weg zum virtuellen Kraftwerk und zur solaren Mobilität . . . . . . . . 156

10.5 Weitere Trends . . . . . . . . . . . . . . . . . . . . . . . . . . 159

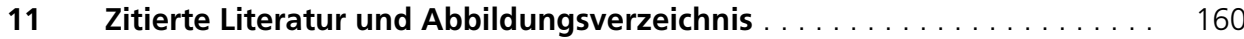

11.1 Zitierte Literatur . . . . . . . . . . . . . . . . . . . . . . . . . . . . . . . . . . . . . 160

11.2 Abbildungsverzeichnis . . . . . . . . . . . . . . . . . . . . . . . . 164

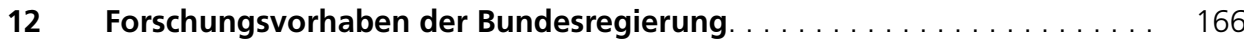

12.1 Laufende und kürzlich abgeschlossene Forschungsvorhaben . . . . . . . . . 166

12.2 Forschungsberichte. . . . . . . . . . . . . . . . . . . . . . . . . . 168

13 Weiterführende Literatur . . . . . . . . . . . . . . . . . . . . . . . . . . 169

13.1 Literatur. . . . . . . . . . . . . . . . . . . . . . . . . . . . . . 169

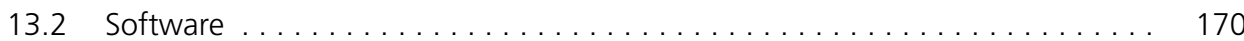

13.3 Literaturtipp . . . . . . . . . . . . . . . . . . . . . . . . . . . . . . . . 170

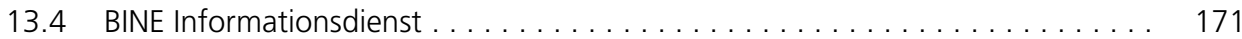

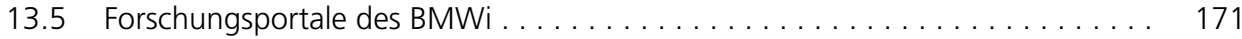

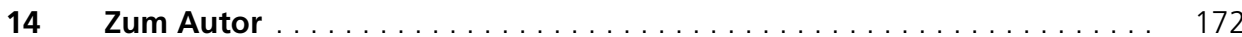




\section{Vorwort}

Überall im Land gehören Dächer mit Photovoltaik-Anlagen zum Ortsbild. Die Zahl der Anlagen wächst weiter, für die Besitzer sind sie zugleich eine sinnvolle Investition und ein Statement für umweltgerechte Energieerzeugung. Der zukunftsfähige Wirtschaftszweig nachhaltige Energieerzeugung reduziert Ressourcenverbrauch und Emissionen und schafft Einkommen und Arbeit. Die Energiebilanz der Photovoltaik ist beeindruckend: Nach zwei Jahren hat ein neues Modul die Energie geliefert, die für seine Produktion gebraucht wurde. Und es wird insgesamt 20-30 Jahre Strom produzieren.

Inzwischen sind Solarstromanlagen mit einer Gesamtleistung von etwa 34 Gigawatt in Deutschland in Betrieb (Stand Juni 2013). Mit der Produktion von 27,6 TWh elektrischer Energie im Jahr 2012 deckte die Photovoltaik etwa 5,3\% des Nettostromverbrauchs. Photovoltaik bleibt trotz jährlich sinkender Einspeisevergütungen und Förderobergrenzen interessant. Das neue Ziel ist es, einen hohen Eigenverbrauch zu erreichen - das entlastet Kasse und Stromnetz. Deshalb investieren gegenwärtig insbesondere Hausbesitzer in neue Anlagen, die durch höhere Eigenverbrauchsquoten den stark abgesenkten Einspeisetarif zum Teil ausgleichen können.

Der Boom der Solarenergie wurde im Jahr 2000 durch das Erneuerbare-Energien-Gesetz (EEG) angestoßen. Durch die Förderung der Einspeisung von Strom aus erneuerbaren Quellen ermöglichte es enorme Fortschritte bei Forschung, Entwicklung und Produktion von Solarzellen, Modulen und anderen Anlagenkomponenten, z. B. Wechselrichtern. Die Forschungs- und Entwicklungsarbeit der deutschen Solarbranche wird von der Bundesregierung auch im Rahmen der Innovationsallianz Photovoltaik unterstützt. Dank effizienterer und kostengünstigerer Herstellungsprozesse kann (und muss) die Industrie von Jahr zu Jahr leistungsfähigere und günstigere Photovoltaikmodule und -anlagen anbieten.

Die Anlagenpreise sinken nicht nur durch technischen Fortschritt, sie stehen zusätzlich durch internationale Konkurrenz sowie Deckelung des Zubaus unter Druck. Im ersten Halbjahr 2013 wurde nur noch etwa halb so viel neue PV-Leistung installiert wie im Jahr davor. Die Kombination von Preisdumping und Absatzdeckelung bringt die Zukunftsindustrie Photovoltaik in ernste Schwierigkeiten.

In seiner siebten, völlig überarbeiteten Auflage fasst dieses Fachbuch das aktuelle Wissen für eine optimale Planung und Erstellung von Photovoltaik-Anlagen kompetent und kompakt zusammen. Es richtet sich vor allem an Bauherren, Handwerker, Planer und Investoren, also an die Akteure und Berufsgruppen, die mit der neuen Gebäudetechnik Photovoltaik zu tun haben. Neben den Aktualisierungen werden neue Schwerpunkte gesetzt, zum Beispiel bei Baurecht, Normen und Brandschutz, Eigenverbrauch sowie bei der wirtschaftlichen Bewertung eines Anlagen-Neubaus.

Das Buch ist modular aufgebaut - das macht es Ihnen als Leser einfacher, sich entweder gezielt über einzelne Fragestellungen zu informieren oder sich einen Überblick über Photovoltaik auf Gebäuden zu verschaffen, von Planung und Bau über Wirtschaftlichkeit bis zu aktueller Forschung.

FIZ Karlsruhe GmbH

BINE Informationsdienst 
